\title{
BMJ Open Trends in initiation of direct oral anticoagulant therapies for atrial fibrillation in a national population- based cross-sectional study in the French health insurance databases
}

\author{
Laetitia Huiart, ${ }^{1,2,3,4}$ Cyril Ferdynus, ${ }^{1,2}$ Christel Renoux, ${ }^{5,6,7}$ Amélie Beaugrand, ${ }^{1,8}$ \\ Sophie Lafarge, ${ }^{2}$ Léa Bruneau, ${ }^{1,4}$ Samy Suissa, ${ }^{5,7}$ Olivier Maillard, ${ }^{2,4}$ Xavier Ranouil ${ }^{9}$
}

To cite: Huiart L, Ferdynus C, Renoux C, et al. Trends in initiation of direct oral anticoagulant therapies for atrial fibrillation in a national population-based cross-sectional study in the French health insurance databases. BMJ Open 2018;8:e018180. doi:10.1136/ bmjopen-2017-018180

- Prepublication history and additional material for this paper are available online. To view these files, please visit the journal online (http://dx.doi org/10.1136/bmjopen-2017018180).

Received 12 June 2017 Revised 12 December 2017 Accepted 25 January 2018

Check for updates

For numbered affiliations see end of article.

Correspondence to Professor Laetitia Huiart; laetitia.huiart@mail.mcgill.ca

\section{ABSTRACT}

Objective Unlike several other national health agencies, French health authorities recommended that the newer direct oral anticoagulant (DOAC) agents only be prescribed as second choice for the treatment of newly diagnosed nonvalvular atrial fibrillation (NVAF), with vitamin $\mathrm{K}$ antagonists (VKA) remaining the first choice. We investigated the patterns of use of DOACs versus VKA in the treatment of NVAF in France over the first 5 years of DOAC availability. We also identified the changes in patient characteristics of those who initiated DOAC treatment over this time period.

Methods Based on the French National Health Administrative Database, we constituted a populationbased cohort of all patients who were newly treated for NVAF between January 2011 and December 2015. Trends in drug use were described as the percentage of patients initiating each drug at the time of treatment initiation. A multivariate analysis using logistic regression model was performed to identify independent sociodemographic and clinical predictors of initial anticoagulant choice.

Results The cohort comprised 814446 patients who had received a new anticoagulant treatment for NVAF. The proportion of patients using DOACs as initial anticoagulant therapy reached $54 \% 3$ months after the Health Ministry approved the reimbursement of dabigatran for NVAF, and $61 \%$ by the end of 2015 , versus VKA use. In the multivariate analysis, we found that DOAC initiators were younger and healthier overall than VKA initiators, and this tendency was reinforced over the 2011-2014 period. DOACs were more frequently prescribed by cardiologists in 2012 and after (adjusted OR in 2012: 2.47; 95\% Cl 2.40 to 2.54).

Conclusion Despite recommendations from health authorities, DOACs have been rapidly and massively adopted as initial therapy for NVAF in France. Observational studies should account for the fact that patients selected to initiate DOAC treatment are healthier overall, as failure to do so may bias the risk-benefit assessment of DOACs.

\section{INTRODUCTION}

Non-valvular atrial fibrillation (NVAF) is the most common sustained cardiac arrhythmia and its age-adjusted prevalence has been

\section{Strength and limitations of this study}

- With a source database covering 66 million inhabitants and exhaustive information on anticoagulant deliveries in France, our study is the largest to report penetration of direct oral anticoagulants (DOACs) on the market.

- The extensive size of the cohort comprising 814446 individuals provides reliable information even for the most recent DOAC available at the time of the study, apixaban.

- The administrative database used does not include clinical results nor does it include outpatients' diagnosis codes. To account for outpatients, we based our definition of non-valvular atrial fibrillation (NVAF) on drug dispensation, using the most likely treatment scheme for NVAF. We conducted sensitivity analyses to ensure that our results are consistent.

increasing over time. ${ }^{1}{ }^{2}$ NVAF is associated with high morbidity and mortality, as it increases the risks of stroke and systemic thromboembolic events. ${ }^{2}$ In light of this, the use of an oral anticoagulant is recommended in patients with NVAF at medium or high risk of stroke. ${ }^{3-7}$ For more than 50 years, vitamin $\mathrm{K}$ antagonists (VKA), such as warfarin, were the only effective therapy available for stroke prevention in patients with NVAF. ${ }^{8}$ However, the efficacy and safety of VKAs are closely related to the quality of anticoagulation, which is open to substantial interpatient and intrapatient variability and requires close biological monitoring. ${ }^{8-10}$

To respond to physicians' and patients' expectations of more user-friendly drugs, research on new drugs has intensified over the last few years. This has prompted the development of direct oral anticoagulants (DOAC) that inhibit thrombin or factor Xa. 
Large randomised clinical trials (RCTs) have compared the efficacy of DOACs with that of warfarin in patients with NVAF. ${ }^{11-13}$ A recent meta-analysis of these RCTs has shown that the frequency of strokes and/or systemic embolic events is $19 \%$ lower with DOACs than it is with warfarin. Moreover, compared with warfarin, DOACs have been shown to present similar risks of major bleeding but higher risks of gastrointestinal bleeding. ${ }^{14}$ The benefitrisk ratio of DOACs nevertheless varies across individual agents and according to patient's profile. ${ }^{1516}$

In France, the direct thrombin inhibitor dabigatran was the first DOAC to be approved for the primary prevention of venous thromboembolism in orthopaedic surgery (in 2008) and stroke in NVAF (in 2011). Reimbursement of dabigatran for the treatment of NVAF was approved in July 2012. Two new oral direct factor Xa inhibitors, rivaroxaban and apixaban, were made available for patients with NVAF in September 2012 and January 2014, respectively. Given the initial lack of specific antidotes and of data on these drugs' efficacy and safety in real life, French health authorities recommended that VKAs remain the standard therapy. They also recommended that DOACs be offered as an alternative therapy only to patients with low adherence to VKAs or unstable international normalised ratios (INRs) on VKAs. ${ }^{17}$ To date, it is not clear how the expectations of clinicians and the recommendations of health authorities have impacted the choice of anticoagulant for newly treated patients with NVAF. Nor is it clear how patients' characteristics have influenced treatment choice.

In view of the above, we conducted a study in the French National Health administrative database. This study based on claims data, aimed to identify the initial oral anticoagulant therapy used in a cohort of patients newly diagnosed with NVAF for the prevention of stroke and systemic thromboembolism. It also sought to describe changes in the characteristics of patients who initiated treatment during the first 5 years of DOAC availability in France.

\section{METHOD}

\section{Study design and source of data}

The retrospective population-based cohort of patients with NVAF was formed from data provided by the French National Health Insurance System (NHIS) ${ }^{18}$ The NHIS guarantees universal health coverage to all segments of the population and includes both a drug delivery database and a hospital discharge database. The NHIS comprises health insurance schemes for salaried workers, self-employed workers, agricultural workers and farmers, as well as 12 other insurance schemes. Together, these schemes provide health insurance to approximately 66 million inhabitants, which corresponds to approximately $99 \%$ of the French population. ${ }^{19}$ Detailed description of the NHIS database is provided elsewhere. ${ }^{20} 21$

In France, drugs are available only in pharmacies, and a medical prescription is required to obtain anticoagulant drugs. All reimbursement claims for prescriptions processed and filled in pharmacies are submitted to the NHIS via a single electronic system. This drug delivery database is linked to the hospital discharge database through a unique personal identifier allocated to every individual. The second database provides medical information on all patients discharged from hospitals, along with associated 10th version of International Classification of Diseases diagnosis codes. However, no clinical diagnosis is provided in this database for consultations by health professionals in an ambulatory care setting.

\section{Cohort definition}

We defined a cohort of all patients 18 years and older who were newly treated for NVAF between 1 January 2011 and 31 December 2015. Cohort entry was defined by the delivery of anticoagulant therapy (VKA or DOAC) combined with either an antiarrhythmic agent (flecainide, propafenone, amiodarone, quinidine, disopyramide or sotalol) or a rate control treatment (beta-blocker, calcium channel blockers-verapamil and diltiazem-or digoxin) within a time window of $+/-30$ days. The date of cohort entry was the latest date of delivery of either drug, within the 30-day window. We excluded patients with less than 1year of data available in the database before cohort entry, as well as patients who had received anticoagulant treatment or had a history of cardiac valvular replacement in the 12 months before inclusion. Therefore, the anticoagulant therapy received at cohort inclusion corresponded to a new anticoagulant therapy. Lastly, we excluded patients who had undergone lower limb orthopaedic surgery within $+/-30$ days of inclusion.

\section{Exposure}

We identified patients' exposure to initial anticoagulant treatment. We compared patients initiating VKA treatment-acenocoumarol, fluindione, warfarin (the three most commonly used VKAs in France)-to patients receiving any of the three DOACs available during the study period (dabigatran, rivaroxaban or apixaban).

\section{Study covariates}

The following characteristics of patients were identified in the year prior to cohort entry using treatment and/ or hospital discharge code (see online supplementary table): high blood pressure, coronary artery disease (including myocardial infarction and ischaemic heart disease), congestive heart failure, diabetes, a personal history of cancer, renal failure, liver failure, dementia, a history of bleeding and history of ischaemic stroke. Exposure to treatment other than anticoagulants-aspirin or other non-steroidal anti-inflammatory drugs (NSAIDs), antiplatelet agents (other than aspirin), corticosteroidswas identified in 3 months prior to cohort entry. We also determined whether initial anticoagulant therapy was prescribed by a general practitioner, a cardiologist or a physician with another specialty. To estimate the risk of major bleeding, we calculated a modified HAS-BLED 
score (hypertension, abnormal renal and/or liver function, stroke/transient ischaemic attack, bleeding, labile INR, age $>65$, antiplatelet/NSAID use or alcohol abuse) ${ }^{22}$ Labile INR was not included in the score because it is unavailable in the database. Alcohol abuse was determined based on the hospital discharge database. To estimate the risk of stroke, we calculated the $\mathrm{CHA}_{2} \mathrm{DS}_{2}$-VASc score (congestive heart failure, hypertension, age $\geq 75$, diabetes mellitus, stroke/transient ischaemic attack, vascular disease, age $65-74$ and sex). ${ }^{23}$

\section{Data analysis}

Descriptive statistics were computed for continuous data (mean,+/-SD or median and range) and for categorical data (frequency and proportion). Trends in drugs use were described as the number of new patients treated each month and as the percentage of each anticoagulant prescribed at the time of treatment initiation.

Patients' characteristics were described according to initial anticoagulant therapy received. In bivariate comparisons, the characteristics of patients and prescribers were compared according to the type of anticoagulant, using a t-test for continuous variables and a $\chi^{2}$ test for categorical variables. To identify independent predictors of initial anticoagulant choice, we performed a multivariate analysis using five logistic regression models, one for each calendar year of anticoagulant initiation. The model included all the variables that were associated with a $p$ value $<0.20$ in the bivariate comparisons. These variables were selected using a backward selection approach. Further, we defined two other cohorts for the sensitivity analyses: (1) one cohort was defined more restrictivelyit included patients who were newly treated with an anticoagulant combined with an antiarrhythmic agent or a rate control treatment other than beta-blocker agents within a time window of +/-30 days; (2) the other cohort was defined according to broader inclusion criteria-it comprised all patients newly treated with an anticoagulant, regardless of other potential concomitant therapies.

To assess the impact of timeline events on the uptake process (ie, market authorisation of each drug, reimbursement approval/downgrade and security warnings from national health agency), we fitted a segmented regression model adjusted on: (1) drug coded into four categories (VKA, dabigatran, rivaroxaban and apixaban); (2) time (linear and square terms) and (3) each timeline event. A timeline event was coded as a dichotomous variable valued 0 before the event and 1 after. All these covariates were included in a primary model, then a backward selection procedure was applied to select covariates associated at a significant level $(\mathrm{p}<0.05)$. To evaluate the trends and the impact of timeline events on each drug, we entered an interaction term for each drug and other covariates (time and timeline events).

Statistical significance was set at 0.05 . All $p$ values were two sided.

All statistical analyses were performed using SAS V.9.4 (SAS institute).

\section{RESULTS}

In France, 1772399 individuals were delivered a prescription for either a VKA or a DOAC between 1 January 2011 and 31 December 2015 (figure 1). Out of this sample, we identified 872970 individuals who received a new prescription for an anticoagulant (VKA or DOAC) combined with a prescription for an antiarrhythmic agent or a rate control treatment within a time window of $+/-30$ days. Ultimately, the cohort comprised 814446 patients newly treated for NVAF: 506821 subjects initiated VKA, 94468 dabigatran, 169524 rivaroxaban and 43633 apixaban.

Figure 2 illustrates the percentages of patients initiating one of the different anticoagulant treatments over the study period. A sharp rise in DOAC use was observed starting in mid-2012. As of October 2012, DOACs were used more frequently than VKAs as initial anticoagulant therapy, representing $54 \%$ of all anticoagulant prescriptions (30\% for dabigatran and $24 \%$ for rivaroxaban). In the last quarter of 2015, this percentage reached $61 \%$ ( $4 \%$ for dabigatran, $34 \%$ for rivaroxaban and $24 \%$ for apixaban). The proportion of patients initiating dabigatran began to decline 6 months after reimbursement was approved and even more so after October 2013. Rivaroxaban use increased sharply as early as September 2012. This drug was the most frequently initiated DOAC in early 2013, and it remained so until December 2015, when it was surpassed by apixaban (26\% vs $28 \%$ in December 2015).

Figure 2 illustrates the percentages of patients initiating one of the different anticoagulant treatments over the study period. The segmented regression model identified five significant change points. The two first change points (lines $\mathrm{a}$ and $\mathrm{b}$ in figure 2) corresponded to a sharp rise in DOAC initiation in July 2012 and in September 2012, corresponding respectively to dabigatran and rivaroxaban reimbursement approval time. As of October 2012, DOACs were used more frequently than VKAs as initial anticoagulant therapy, representing $54 \%$ of all anticoagulant prescriptions $(30 \%$ for dabigatran and $24 \%$ for rivaroxaban). The third change point identified was in September 2013 (line d, figure 2) with a significant decrease in the use of DOACs at the time security warnings were issued by the French health authorities. From January 2014 (fourth change point-line c on figure 2), DOACs initiation increased again, corresponding to the time point where apixaban received reimbursement approval. A final significant change point (line e) was identified in September 2015 and was linked to a reduction in dabigatran reimbursement. In December 2015, apixaban was the most prescribed DOAC (28\% vs $26 \%$ for rivaroxaban).

The mean age of newly treated patients was 74.9 (SD: 11.7 ), and $50.2 \%$ of patients were male (table 1 ). Most subjects were treated for high blood pressure $(94.4 \%)$, and $22.2 \%$ were treated for diabetes. Patients who received DOACs had less comorbidities and were on average younger than those who were prescribed 


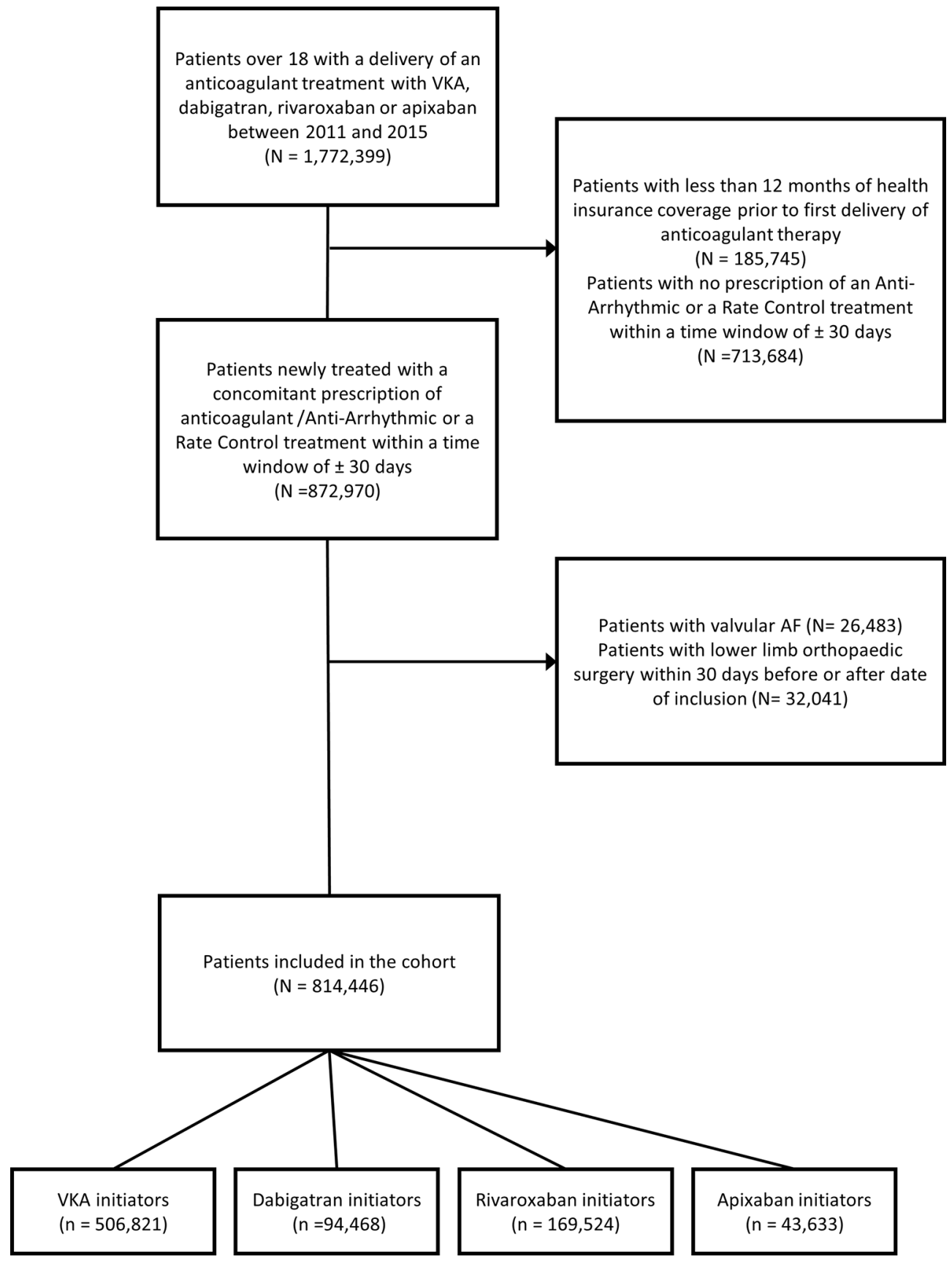

Figure 1 Flowchart describing cohort constitution. $\mathrm{AF}$, atrial fibrillation; VKA, vitamin $\mathrm{K}$ antagonist.

VKAs (73.8 years (SD: 11.5) vs 75.6 years (SD: 11.9) $\mathrm{p}<0.0001)$. General practitioners prescribed VKAs $(67.5 \%)$ more frequently than DOACs $(32.5 \%)$ as initial anticoagulant therapy, whereas cardiologist favoured DOACs $(51.2 \%)$. Patients receiving apixaban were older than those receiving other DOACs (76.2 vs 72.9 for rivaroxaban and 74.1 for dabigatran). They also had more comorbidities, such as high blood pressure and heart or renal failure (table 1). Patients with lower HAS-BLED or lower $\mathrm{CHA}_{2} \mathrm{DS}_{2}$-VASc scores were more likely to initiate DOACs (figure 3 ).

The characteristics and associated treatments of DOAC initiators as compared with VKA initiators changed over the 5-year period (table 2). Older subjects $(>=75$ years) were less likely to initiate DOAC treatment than VKA treatment. The adjusted OR decreased from 0.86 in 2011 (95\% CI 0.80 to 0.92 ) to 0.62 in 2014 (95\% CI 0.61 to 0.64 ). Overall, patients with comorbidities-especially renal failure-were less likely to receive DOAC treatment, and this negative association was reinforced over the study period (adjusted OR for 2014: 0.22 ; 95\% CI 0.21 to 0.23 ). The negative association was not reinforced in 2015, likely due the fact that a larger proportion of patients received apixaban. However, because apixaban was only available at the end of the study period, further data are needed to confirm this hypothesis. Patients with a history of bleeding prior to cohort entry were 
(a) (b) (d) (c)

(e)

Figure 2 Time trends in the prescription of anticoagulants in newly treated patients with atrial fibrilation between 2011 and 2015 in France $(n=814446)$. Significant change points in trends identified a segmented regression model. (a) Dabigatran reimbursement approval, (b) rivaroxaban reimbursement approval (c) and apixaban reimbursement approval, (d) security warning (risks of bleeding haemorrhages) from the national health agency, (e) downgrade of dabigatran reimbursement. DOACs, direct oral anticoagulants; VKA, vitamin $\mathrm{K}$ antagonist.

less likely to receive DOAC treatment (adjusted OR for 2015: 0.56 ; $95 \%$ CI 0.53 to 0.59 ). Since 2012 , cardiologists have been strongly associated with initial prescription of DOACs, after accounting the patients' characteristics.

The sensitivity analyses conducted for the two other cohorts provided similar results: a rapid increase in the use of DOAC over time, and a healthier profile of patients receiving DOACs compared with those receiving VKAs (see online supplementary material cohort 1 and 2).

\section{DISCUSSION}

Less than 6 months after reimbursement was approved, DOACs became the most frequently prescribed initial anticoagulant therapy for NVAF in France. Starting in the third quarter of 2012, DOACs were delivered to over $60 \%$ of all patients newly treated for NVAF. Dabigatran, rivaroxaban and, in late 2015, apixaban were used one after the other as the most frequent initial DOAC therapy for NVAF. The proportion of dabigatran initiators declined largely after 2013. DOAC initiators were younger and 
Table 1 Characteristics of patients and prescribers at anticoagulant treatment initiation (2011-2015)

\begin{tabular}{|c|c|c|c|c|}
\hline & $\begin{array}{l}\text { VKA } \\
\mathrm{N}=506821\end{array}$ & $\begin{array}{l}\text { Dabigatran } \\
\mathrm{N}=94468\end{array}$ & $\begin{array}{l}\text { Rivaroxaban } \\
\mathrm{N}=169524\end{array}$ & $\begin{array}{l}\text { Apixaban } \\
\mathrm{N}=43633\end{array}$ \\
\hline \multicolumn{5}{|l|}{ Demographic characteristics } \\
\hline Mean age (SD) & $75.6(11.9)$ & $74.1(11.3)$ & $73.0(11.5)$ & $76.2(11.1)$ \\
\hline Male & $49.3 \%$ & $52.3 \%$ & $52.0 \%$ & $49.5 \%$ \\
\hline \multicolumn{5}{|l|}{ Clinical characteristics ${ }^{*}$} \\
\hline High blood pressure & $95.4 \%$ & $92.1 \%$ & $92.5 \%$ & $94.7 \%$ \\
\hline Ischaemic heart disease & $28.6 \%$ & $19.7 \%$ & $17.4 \%$ & $17.6 \%$ \\
\hline Heart failure & $27.8 \%$ & $18.9 \%$ & $15.2 \%$ & $21.5 \%$ \\
\hline Diabetes & $23.6 \%$ & $19.9 \%$ & $19.7 \%$ & $20.8 \%$ \\
\hline Cancer & $16.5 \%$ & $14.0 \%$ & $12.8 \%$ & $11.1 \%$ \\
\hline Renal failure & $10.9 \%$ & $2.3 \%$ & $2.4 \%$ & $4.1 \%$ \\
\hline Liver failure & $1.7 \%$ & $0.7 \%$ & $0.7 \%$ & $0.6 \%$ \\
\hline Dementia & $5.2 \%$ & $3.1 \%$ & $2.9 \%$ & $3.3 \%$ \\
\hline History of ischaemic stroke & $9.6 \%$ & $8.4 \%$ & $6.0 \%$ & $9.0 \%$ \\
\hline History of bleeding & $6.3 \%$ & $2.9 \%$ & $3.1 \%$ & $3.9 \%$ \\
\hline HAS-BLED score, mean (SD) & $2.7(0.9)$ & $2.4(0.9)$ & $2.4(0.9)$ & $2.5(0.9)$ \\
\hline $\mathrm{CHA}_{2} \mathrm{DS}_{2}-\mathrm{VASc}$, mean (SD) & $3.9(1.5)$ & $3.5(1.5)$ & $3.3(1.4)$ & $3.7(1.4)$ \\
\hline \multicolumn{5}{|l|}{ Other treatments at cohort entry $\dagger$} \\
\hline Aspirin & $45.8 \%$ & $43.3 \%$ & $40.9 \%$ & $43.6 \%$ \\
\hline NSAIDs & $13.7 \%$ & $16.6 \%$ & $16.9 \%$ & $13.0 \%$ \\
\hline Antiplatelet agents (other than aspirin) & $15.8 \%$ & $12.1 \%$ & $10.9 \%$ & $12.4 \%$ \\
\hline Corticosteroids & $14.0 \%$ & $12.2 \%$ & $12.7 \%$ & $12.1 \%$ \\
\hline Protons-pump inhibitors & $48.9 \%$ & $40.7 \%$ & $41.2 \%$ & $43.9 \%$ \\
\hline \multicolumn{5}{|l|}{ Prescriber of first anticoagulant } \\
\hline General practitioner & $64.4 \%$ & $50.2 \%$ & $51.9 \%$ & $50.4 \%$ \\
\hline Cardiologist & $22.2 \%$ & $38.9 \%$ & $38.0 \%$ & $37.9 \%$ \\
\hline Other specialist & $4.8 \%$ & $4.4 \%$ & $4.7 \%$ & $4.6 \%$ \\
\hline Unknown & $8.6 \%$ & $6.5 \%$ & $5.4 \%$ & $7.1 \%$ \\
\hline
\end{tabular}

${ }^{*} D$ efined in the 12 months prior to cohort entry.

†Defined in the 3 months prior to cohort entry.

NSAIDs, non-steroidal anti-inflammatory drugs; SD, standard deviation; VKA, vitamin K antagonist.

healthier than VKA users, and this tendency was reinforced over time. The use of DOACs varied over time depending on the availability of new drugs and on the national recommendations and safety warnings in place.

National trends in anticoagulant sales volumes have also been reported in other countries, revealing an important upsurge in DOAC use. ${ }^{24-26}$ Most studies based on registries or cohorts of patients with NVAF have reported dabigatran use rates ranging from $5 \%$ to $25 \%$, which is lower than what we found in our study. ${ }^{27-30}$ However, in the USA, Desai et al have reported an increase in DOAC use for the 2010-2013 period which is similar to the one we observed, with a peak of $62 \%$ of patients initiating DOAC treatment among a cohort of anticoagulant initiators. ${ }^{31}$ These common trends in results observed are surprising given the differences in populations, health systems, drug coverage and, most importantly, clinical recommendations on the use of DOACs for the treatment of NVAF between countries. Indeed, in France, health authorities do not recommend DOACs as initial anticoagulant therapy, unless the patient has poor adherence to VKAs or unless biological monitoring of VKA treatment is difficult. ${ }^{17}$ However, physicians are still free to opt for any of the available treatment and their personal beliefs on efficacy and safety influences their choices.

The sharp rise in DOAC use in France was observed as of mid-2012, shortly after the NHIS approved the reimbursement of dabigatran. Specifically, dabigatran was authorised as an anticoagulant for the treatment of NVAF in August 2011; reimbursement of treatment was preapproved in February 2012, and it was fully approved in July 2012. Starting in November 2012, DOACs were used more frequently than VKAs as initial anticoagulant therapy. The reimbursement of rivaroxaban was fully 


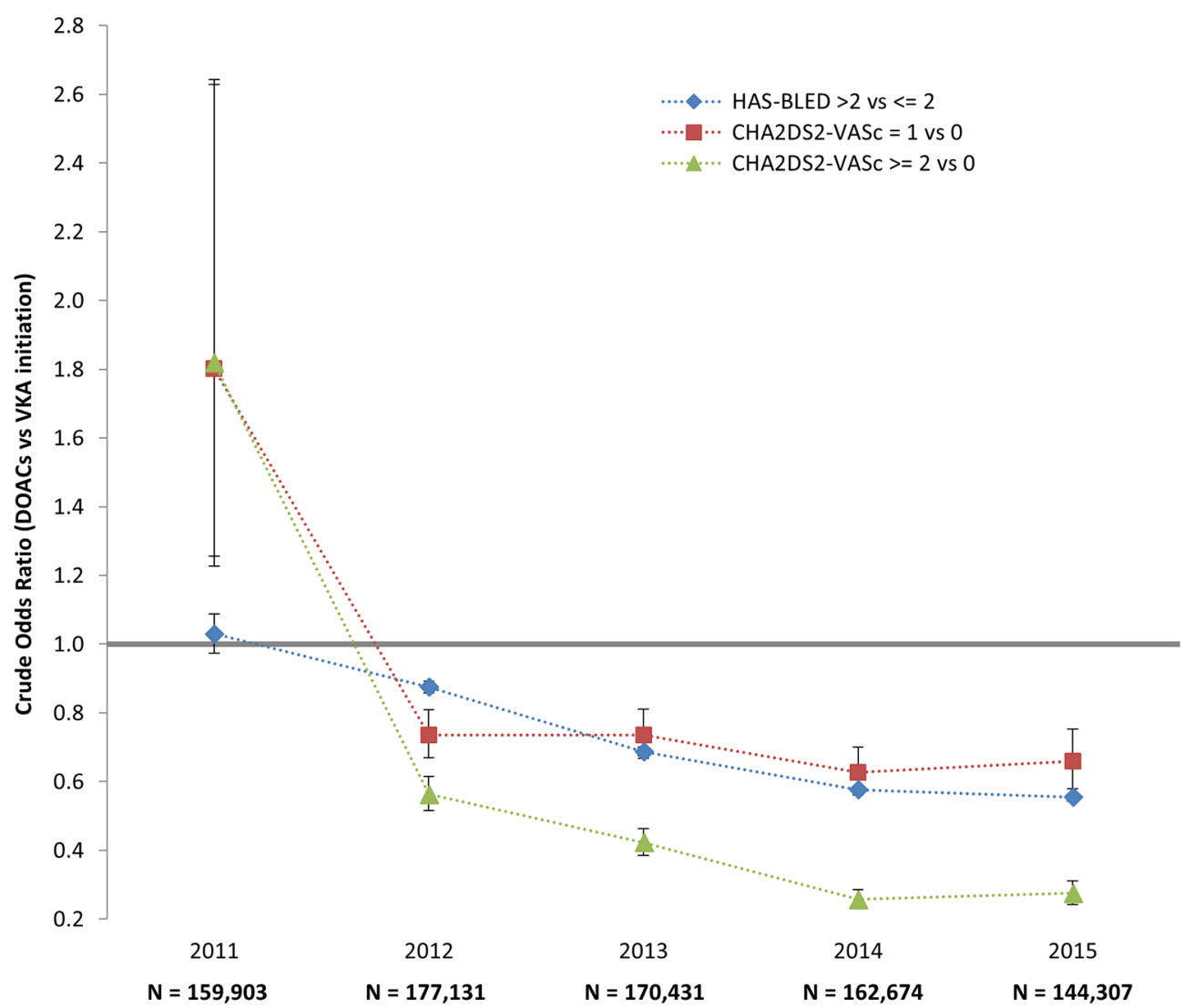

Figure 3 HAS-BLED and CHA DS2-VASc scores associated with DOAC vs VKA initiation according to year of therapy initiation. Crude ORs and $95 \% \mathrm{Cl}$. DOAC, direct oral anticoagulant ; VKA, vitamin K antagonist.

approved in September 2012, and the drug was used more frequently than dabigatran as initial anticoagulant therapy as of January 2013. This rapid uptake of DOACs in the drug market may be explained by the fact that alternatives to VKAs had long been expected. Indeed, a recent study indicates that DOACs were considered equal or preferred to VKAs by respectively $48.5 \%$ and $33.3 \%$ of surveyed physicians. ${ }^{32}$ The speed of adoption of DOACs is similar to that described for other new drugs, which usually reaches a plateau 6 to 12 months after they are launched. ${ }^{33}$ This speed varies according to the specialty of the prescriber, and specialists are generally more prompt to adopt new drugs ${ }^{33}$ - as was the case in our study. Nevertheless, some studies have reported no impact of physician specialty on the prescription of DOACs. ${ }^{27} 30$ The differences we observed between the prescriptions of general practitioners (GP) and those of cardiologists may reflect the gap between national and European clinical guidelines. Indeed, French Health Authorities recommend VKAs as initial anticoagulant therapy, whereas the European Society of Cardiology favours DOACs. ${ }^{3}$ GPs in the rest of Europe have taken a more cautious approach towards DOACs. This is especially the case in the treatment of elderly populations, most likely because there remains substantial uncertainty concerning the effectiveness and safety of DOACs in unselected elderly patients with NVAF. ${ }^{34}$
Our results indicate that the characteristics of patients who initiated treatment with DOACs rather than VKAs evolved over the first few years of drug commercialisation. In the first year, we observed a selection process with healthier patients using DOACs more frequently than VKAs as initial therapy. ${ }^{31}$ This tendency was reinforced as DOAC initiators became healthier over time. It may reflect the evolution of the perception of efficacy and safety of these new drugs by physicians. The prescription of DOACs to healthier patients is an issue that needs to be addressed, as these molecules may offer higher risk patients greater benefits than VKAs, ${ }^{35}$ and because their cost-effectiveness depends on the severity of patients' condition. ${ }^{36}$ Observational studies that aim to evaluate the risks and benefits associated with DOACs as well as cost-effectiveness studies should carefully account for the fact that patients selected to initiate DOAC treatment are healthier overall, as well as for the selection of patients on the different types of DOACs. ${ }^{37}$ Failure to do so may lead to underestimating the potential risks associated with DOACs in real-life studies.

The fact that DOAC initiation is less frequent among patients with comorbidities may result from a warning issued by different health agencies such as in France, Europe or USA. ${ }^{35}$ This tendency seems to be linked to the diminishing use of dabigatran observed at the end of 2013, when the French medicine safety agency released warnings 


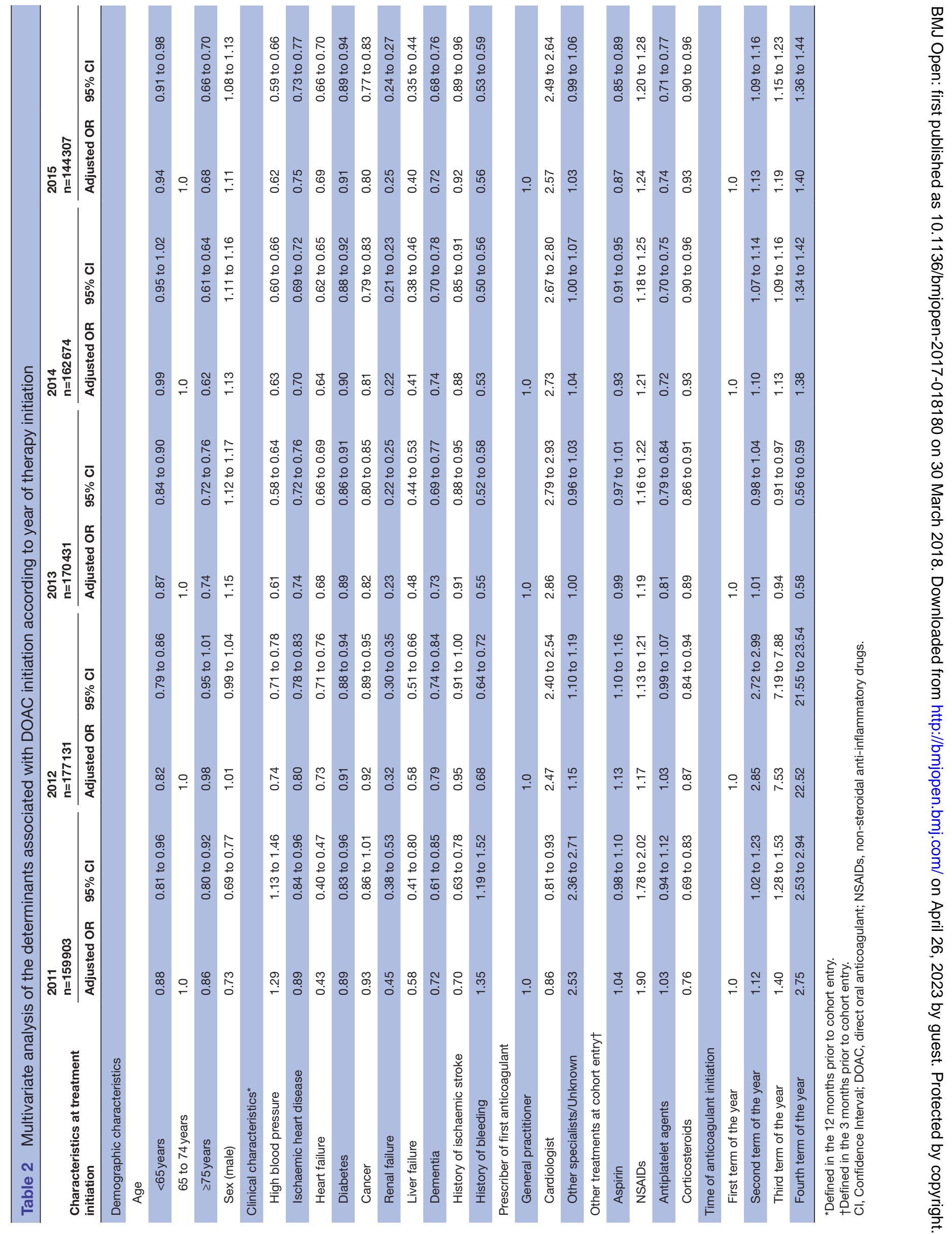


on bleeding risks associated with the drug. ${ }^{38-40}$ At the time, French health authorities informed health professionals that DOACs are not recommended as initial therapy for NVAF, unless the patient has poor adherence to VKAs or unless biological monitoring of VKA treatment is difficult. However, while these recommendations were followed by a temporary decrease in DOAC use, a few months later, DOACs were once again the most frequently prescribed anticoagulants for patients newly treated for NVAF.

Our study has several strengths. The source database covers 66 million inhabitants and nearly $99 \%$ of the French population, which means that our findings are independent of individual health coverage. Moreover, we had access to exhaustive information on anticoagulant delivery because these treatments are delivered on prescription alone. As a result, our study is the largest to report penetration of DOACs on the market (particularly in the case of apixaban, which is the most recent DOAC available) and to describe variations in the characteristics of patients over time.

Nevertheless, some limitations must also be acknowledged. The NHIS administrative database does not include clinical or biological results nor does it include outpatients' diagnosis codes. To capture outpatient diagnosis of AF, we based our definition of NVAF on drug dispensation, using the most likely treatment scheme for NVAF. Insofar as the results of our sensitivity analyses are consistent, we can be confident that our findings regarding the choice of the initial therapy and the patients' characteristics are not too sensitive to the definition of NVAF. Moreover, $69 \%$ of patients who were hospitalised during follow-up had a diagnostic code of NVAF in the hospital discharge database (data not shown). We did not use long duration diseases codes to define AF as these codes have various limitation, for example, their use has been shown to differ between the insurance schemes included in the database, ${ }^{41}$ and there was an important discrepancy between them and hospital discharge codes. These long duration disease codes were only used to define some covariates but only in combination with drugs delivery and/or hospital codes. We did not exclude patients with deep vein thrombosis or pulmonary embolism at inclusion. They represented $4.4 \%$ of the study sample. We conducted a sensitivity analysis excluding these patients and obtained similar results (data not shown). Another limitation of our study is due to the 2015 data that may be partially incomplete. Indeed, for patients who do not have their NHIS card and attend a pharmacy that is not their regular pharmacy - a paper reimbursement form may be issued. The data are then recorded when the paper form is sent to the NHIS and integrated later in the database. When the 2015 data were made available, paper claims were likely to have not been all included. However, this changes the total number of users but not the proportion of users of the different drugs.

The rapid and massive adoption of DOACs as initial therapy for NVAF will impact treatment expenditures because of the important increase in costs associated with these new drugs (in the USA, these costs accounted for more than $90 \%$ of insurer spending on anticoagulants in 2014). ${ }^{31}$ Future observational studies should carefully account for the fact that patients selected to initiate DOAC treatment are healthier overall, and that this tendency is reinforced over the first few years of drug commercialisation. Failure to do so may bias the risk-benefit assessment of DOACs.

\section{Author affiliations}

${ }^{1}$ Unité de Soutien Méthodologique, CHU de la Réunion, Saint-Denis, France

${ }^{2} \mathrm{CHU}$ de la Réunion, INSERM, CIC1410, Saint-Pierre, France

${ }^{3}$ Université de La Réunion, UFR Santé, Saint-Denis, France

${ }^{4}$ INSERM, Université d'Aix-Marseille, IRD, UMR912 "Sciences Économiques et Sociales de la Santé et Traitement de I'Information Médicale" (SESSTIM), Marseille, France

${ }^{5}$ Centre for Clinical Epidemiology, Lady Davis Institute for Medical Research, Jewish General Hospital, Montréal, Canada

${ }^{6}$ Department of Neurology and Neurosurgery, McGill University, Montréal, Canada

${ }^{7}$ Department of Epidemiology and Biostatistics, McGill University, Montréal, Canada ${ }^{8}$ Centre Hospitalier National d'Ophtalmologie des Quinze-Vingts, Paris, France ${ }^{9} \mathrm{CHU}$ de la Réunion, Service de cardiologie, Saint-Denis, France

Acknowledgements We thank the Caisse National de l'Assurance Maladie des Travailleurs Salariés (CNAMTS) and the Institut des Données de Santé (IDS) for their assistance in obtaining the study database. We personally thank Ms Valérie EDEL from the IDS, as well as Mr Laurent DUCHET and Mr Medhi GABBAS from the department in charge of the SNIIRAM DATA at CNAM-TS. We are indebted to Arianne Dorval for proofreading the English manuscript. C Renoux is the recipient of a Chercheur-Boursier award from the Fonds de la recherche du Québe-Santé (FRQS). SS is the recipient of the James McGill Chair.

Contributors LH, XR, CF designed the study. All authors have contributed substantially to the interpretation of results. In addition, $L H$ and $A B$ drafted the article; CF conducted the statistical analysis; CF, CR, SL, LB, SS, OM and XR provided critical revision of the manuscript for important intellectual content. All authors had full access to all of the data (including statistical reports and tables) in the study and can take responsibility for the integrity of the data and the accuracy of the data analysis. All authors approved the version to be published.

Funding This research was supported by a 2012 research grant from the Agence Nationale de Sécurité du Médicament (ANSM).

Competing interests SS has participated in advisory board meetings and received research grants from Bayer, Boehringer Ingelheim, Bristol-Myers-Squibb and Novartis. These activities are conducted outside this submitted study. All other coauthors have no conflict of interest.

Patient consent Not required.

Ethics approval The study was approved by the French National Institute of Data and by the National Commission for Data Protection and Liberties (CNIL-France: authorisation number: 1637014).

Provenance and peer review Not commissioned; externally peer reviewed.

Data sharing statement № additional data are available directly from the authors. The datasource of the study is the French National Health Insurance. Data ara available from the Caisse National de l'Assurance Maladie des Travailleurs Salariés (CNAMTS) for academic research.

Open Access This is an Open Access article distributed in accordance with the Creative Commons Attribution Non Commercial (CC BY-NC 4.0) license, which permits others to distribute, remix, adapt, build upon this work non-commercially, and license their derivative works on different terms, provided the original work is properly cited and the use is non-commercial. See: http://creativecommons.org/ licenses/by-nc/4.0/

(C) Article author(s) (or their employer(s) unless otherwise stated in the text of the article) 2018. All rights reserved. No commercial use is permitted unless otherwise expressly granted.

\section{REFERENCES}

1. Lloyd-Jones DM, Wang TJ, Leip EP, et al. Lifetime risk for development of atrial fibrillation: the Framingham Heart Study. Circulation 2004;110:1042-6. 
2. Ball J, Carrington MJ, McMurray JJ, et al. Atrial fibrillation: profile and burden of an evolving epidemic in the 21st century. Int J Cardiol 2013;167:1807-24.

3. Camm AJ, Lip GY, De Caterina R, et al. 2012 focused update of the ESC guidelines for the management of atrial fibrillation: an update of the 2010 ESC guidelines for the management of atrial fibrillationdeveloped with the special contribution of the European Heart Rhythm Association. Europace 2012;14:1385-413.

4. January CT, Wann LS, Alpert JS, et al. 2014 AHA/ACC/HRS guideline for the management of patients with atrial fibrillation: a report of the American College of Cardiology/American Heart Association Task Force on Practice Guidelines and the Heart Rhythm Society. J Am Coll Cardiol 2014;64:e1-76.

5. Jones C, Pollit V, Fitzmaurice D, et al. The management of atrial fibrillation: summary of updated NICE guidance. BMJ 2014;348:g3655

6. Verma A, Cairns JA, Mitchell LB, et al. 2014 focused update of the Canadian Cardiovascular Society Guidelines for the management of atrial fibrillation. Can J Cardiol 2014;30:1114-30.

7. You JJ, Singer DE, Howard PA, et al. Antithrombotic therapy for atrial fibrillation: Antithrombotic Therapy and Prevention of Thrombosis, 9th ed: American College of Chest Physicians Evidence-Based Clinical Practice Guidelines. Chest 2012;141:e531S-75.

8. Hart RG, Pearce LA, Aguilar MI. Meta-analysis: antithrombotic therapy to prevent stroke in patients who have nonvalvular atrial fibrillation. Ann Intern Med 2007;146:857-67.

9. De Caterina R, Husted S, Wallentin L, et al. General mechanisms of coagulation and targets of anticoagulants (Section I). Position Paper of the ESC Working Group on Thrombosis: Task Force on Anticoagulants in Heart Disease. Thromb Haemost 2013;109:569-79.

10. Fihn SD, Callahan CM, Martin DC, et al. The risk for and severity of bleeding complications in elderly patients treated with warfarin. The National Consortium of Anticoagulation Clinics. Ann Intern Med 1996;124:970-9.

11. Connolly SJ, Ezekowitz MD, Yusuf S, et al. Dabigatran versus warfarin in patients with atrial fibrillation. $N$ Engl J Med 2009;361:1139-51.

12. Patel MR, Mahaffey KW, Garg J, et al. Rivaroxaban versus warfarin in nonvalvular atrial fibrillation. N Engl J Med 2011;365:883-91.

13. Granger $\mathrm{CB}$, Alexander JH, McMurray JJ, et al. Apixaban versus warfarin in patients with atrial fibrillation. $N$ Engl J Med 2011;365:981-92.

14. Ruff CT, Giugliano RP, Braunwald E, et al. Comparison of the efficacy and safety of new oral anticoagulants with warfarin in patients with atrial fibrillation: a meta-analysis of randomised trials. Lancet 2014;383:955-62.

15. Potpara TS, Lip GY. Novel oral anticoagulants in non-valvular atrial fibrillation. Best Pract Res Clin Haematol 2013;26:115-29.

16. Shields AM, Lip GY. Choosing the right drug to fit the patient when selecting oral anticoagulation for stroke prevention in atrial fibrillation. J Intern Med 2015;278:1-18.

17. Haute Autorité de Santé. Fibrillation auriculaire non valvulaire: Quelle place pour les anticoagulants oraux non antivitamine K:apixaban (Eliquis $®$ ), dabigatran (Pradaxa $®$ ) et rivaroxaban (Xarelto $®$, 2013. http://www.has-sante.fr/portail/upload/docs/application/pdf/ 201307/fs_bum_naco_v5.pdf.

18. Ferdynus C, Huiart L. [Technical improvement of cohort constitution in administrative health databases: Providing a tool for integration and standardization of data applicable in the French National Health Insurance Database (SNIIRAM)]. Rev Epidemiol Sante Publique 2016;64:263-9.

19. Bezin J, Duong M, Lassalle R, et al. The national healthcare system claims databases in France, SNIIRAM and EGB: Powerful tools for pharmacoepidemiology. Pharmacoepidemiol Drug Saf 2017;26:954-62.

20. Martin-Latry K, Bégaud B. Pharmacoepidemiological research using French reimbursement databases: yes we can!. Pharmacoepidemiol Drug Saf 2010;19:256-65.

21. Palmaro A, Moulis G, Despas F, et al. Overview of drug data within French health insurance databases and implications for pharmacoepidemiological studies. Fundam Clin Pharmacol 2016;30:616-24.

22. Pisters R, Lane DA, Nieuwlaat R, et al. A novel user-friendly score (HAS-BLED) to assess 1-year risk of major bleeding in patients with atrial fibrillation: the Euro Heart Survey. Chest 2010;138:1093-100.

23. Lip GY, Nieuwlaat R, Pisters $R$, et al. Refining clinical risk stratification for predicting stroke and thromboembolism in atrial fibrillation using a novel risk factor-based approach: the Euro Heart Survey on atrial fibrillation. Chest 2010;137:263-72.

24. Kirley K, Qato DM, Kornfield R, et al. National trends in oral anticoagulant use in the United States, 2007 to 2011. Circ Cardiovasc Qual Outcomes 2012;5:615-21.

25. Barnes GD, Lucas E, Alexander GC, et al. National trends in ambulatory oral anticoagulant use. Am J Med 2015;128:1300-5.

26. Loo SY, Dell'Aniello S, Huiart L, et al. Trends in the prescription of novel oral anticoagulants in UK primary care. Br J Clin Pharmacol 2017;83:2096-106.

27. Steinberg BA, Holmes DN, Piccini JP, et al. Early adoption of dabigatran and its dosing in US patients with atrial fibrillation: results from the outcomes registry for better informed treatment of atrial fibrillation. J Am Heart Assoc 2013;2:e000535.

28. Sørensen R, Gislason G, Torp-Pedersen C, et al. Dabigatran use in Danish atrial fibrillation patients in 2011: a nationwide study. BMJ Open 2013;3:e002758.

29. Lip GY, Laroche C, Dan GA, et al. 'Real-world' antithrombotic treatment in atrial fibrillation: The EORP-AF pilot survey. Am J Med 2014;127:519-29.

30. Mochalina N, Jöud A, Carlsson M, et al. Antithrombotic therapy in patients with non-valvular atrial fibrillation in Southern Sweden: a population-based cohort study. Thromb Res 2016;140:94-9.

31. Desai NR, Krumme AA, Schneeweiss $S$, et al. Patterns of initiation of oral anticoagulants in patients with atrial fibrillation- quality and cost implications. Am J Med 2014;127:1075-82.

32. Larsen TB, Potpara T, Dagres N, et al. Preference for oral anticoagulation therapy for patients with atrial fibrillation in Europe in different clinical situations: results of the European Heart Rhythm Association Survey. Europace 2015;17:819-24.

33. Garjón FJ, Azparren A, Vergara l, et al. Adoption of new drugs by physicians: a survival analysis. BMC Health Serv Res 2012;12:56.

34. Opstelten W, van den Donk M, Kuijpers T, et al. New oral anticoagulants for nonvalvular atrial fibrillation in the elderly: limited applicability in primary care. Eur J Gen Pract 2015;21:1-5.

35. Lip GY, Lane DA. Stroke prevention in atrial fibrillation: a systematic review. JAMA 2015;313:1950-62.

36. Wisløff T, Hagen G, Klemp M. Economic evaluation of warfarin, dabigatran, rivaroxaban, and apixaban for stroke prevention in atrial fibrillation. Pharmacoeconomics 2014;32:601-12.

37. Schneeweiss S, Gagne JJ, Glynn RJ, et al. Assessing the comparative effectiveness of newly marketed medications: methodological challenges and implications for drug development. Clin Pharmacol Ther 2011;90:777-90.

38. Agence Nationale de Sécurité du Médicament et des produits de santé. Nouveaux anticoagulants oraux Eliquis (apixaban), Pradaxa (dabigatran), Xarelto (rivaroxaban): mise en garde sur les facteurs de risques hémorragiques - Lettre aux professionnels de santé, 2013.

39. Haute Autorité de Santé. Les anticoagulants oraux antivitamine $K$ restent la référence dans la fibrillation auriculaire non valvulaire Communiqué de presse, 2013.

40. Haute Autorité de Santé. Point sur l'utilisation des nouveaux anticoagulants oraux - Communiqué de Presse. 2013 http://www. has-sante.fr/portail/jcms/c_1700943/fr/point-sur-I-utilisation-desnouveaux-anticoagulants-oraux.

41. Tuppin P, Rudant J, Constantinou P, et al. Value of a national administrative database to guide public decisions: From the système national d'information interrégimes de l'Assurance Maladie (SNIIRAM) to the système national des données de santé (SNDS) in France. Rev Epidemiol Sante Publique 2017;65 Suppl 4(Suppl 4):S149-S167. 